ISSN electrónico: 2172-9077

https://doi.org/10.14201/fjc201715127144

\title{
DISCURSOS Y PRÁCTICAS EN TORNO A LAS COMPETENCIAS EN EDUCACIÓN
}

\section{Discourses and Practices around the Competencies in Education}

\author{
Dra. Juana M. SANCHO \\ Catedrática de Universidad de la Universidad de Barcelona, España \\ E-mail: jmsancho@ub.edu \\ http//orcid.org/0000-0002-2941-5619
}

Fecha de recepción del artículo: 01/10/2017

Fecha de aceptación definitiva: 18/10/2017

\begin{abstract}
RESUMEN
En este artículo, basado en el conocimiento elaborado a través de distintos proyectos de investigación realizados en los últimos años, en primer lugar, argumento la dificultad de crear discursos y propuestas desde el campo de la educación debido, entre otros factores, a la escasez de medios y recursos para la investigación educativa. En segundo lugar, se sitúa el contexto de los cambios sociales, políticos, económicos, culturales y tecnológicos que han dado lugar a la aparición y expansión, sobre todo desde el campo empresarial, de los discursos sobre la importancia y conveniencia de poner en práctica una visión de la enseñanza basada en las competencias. También discuto las fortalezas, dificultades y peligros que esto conllevan. En tercer lugar, considero las propuestas realizadas por distintos organismos internacionales y su aplicación en el sistema educativo español. A continuación, exploro la dificultad de generar cambios sustantivos en la enseñanza y el aprendizaje, a partir de la promulgación de los reales decretos que estipulan la obligatoriedad de introducir las consideradas competencias básicas en los currículos escolares. El artículo finaliza con algunas conclusiones y propuestas para pensar.
\end{abstract}

Palabras clave: Cambio educativo; competencia digital; competencias sociales y cívicas; condiciones de enseñanza; política educativa; práctica educativa.

\begin{abstract}
In this paper, based on the knowledge elaborated through different research projects carried out in recent years, I first argue the difficulty of creating discourses and proposals from the field of education due, among other factors, to the scarcity of means and resources for educational research. Second, I refer to the social, political, economic, cultural and technological changes that have led to the emergence and expansion, especially from the business field, of the discourses on the importance and convenience of implementing competency-based teaching and learning processes. I also discuss the strengths, difficulties and dangers that this entails. Thirdly, I consider the proposals made by different international organizations and their application in the Spanish education system. Next, I explore the difficulty of generating substantive changes in teaching and learning, from the enactment of the real decrees that stipulate the obligation to introduce the considered basic competences in the school curricula. The article ends with some conclusions and proposals for thinking.
\end{abstract}

Key words: Educational change; digital competence; civic and social competencies; teaching conditions; educational policy; educational practice. 


\section{INTRODUCCIÓN}

Para cada problema complejo hay una respuesta clara, simple y equivocada. H. L. Mencken

La educación, como campo de estudio y de práctica, que afecta al conjunto de las poblaciones, conlleva un nivel de complejidad difícil de encontrar en ninguna otra actividad humana. En un mundo que nos llama a aprender a lo largo y lo ancho de la vida (Banks, Au, Ball, Bell, et al., 2007), la diversidad de los colectivos que necesitan, y en el mejor de los casos logran, acceso a la educación y la formación y el contraste y multiplicidad de intereses y visiones, lo configuran como un ámbito de extremada dificultad. Si a esto le añadimos la persistente falta de recursos para la investigación educativa, entenderemos por qué la educación tiende a adoptar discursos de otros dominios (la Psicología, la Economía, la Empresa...), no como interlocutora, sino como adoptadora.

Lo que resulta paradójico, y para mí inaceptable, es que algo tan fundamental para los individuos, los grupos y la humanidad como un todo, carezca de recursos para analizar en profundidad sus problemas, proponer acciones fundamentadas y articular un sistema permanente de transformación (no de modas pasajeras) orientado a la mejora. Esta falta de interés, no solo no resulta nueva, sino que en países como España ha empeorado en los últimos años. En 1988, Torsten Husén aducía que, en 1971, el presidente del Subcomité Selecto sobre Educación de la Cámara de Representantes de Estados Unidos, señalaba que

alrededor del 10 por ciento de Defensa se dedicaba a investigación y desarrollo y, del de Sanidad, el 4,6 por ciento. "Sin embargo, cuando llegamos a la educación, tan importante la vida de la mente como lo es la defensa de una nación o la salud para un organismo, en todos los niveles educativos encontramos que Estados Unidos gasta un total inferior a un tercio del uno por ciento de sus presupuestos en los procesos de investigación, innovación y renovación planificada" (p. 44).

En 2017, el Gobierno español aumentó en unos 14.000 millones de euros el gasto en Defensa para los próximos siete años, hasta que alcance el 2\% del PIB (Pardo, 2017). Mientras se ha reducido un $41,9 \%$ de los ya exiguos fondos dedicados a la investigación educativa (Álvarez, 2016).

Esta persistente imagen tiende a olvidar la importancia de considerar las aportaciones de todas las disciplinas, pero de forma integrada y situada, en el foco fundamental de la educación: contribuir a un crecimiento individual que permita dar al otro un lugar en la propia vida (Gert Biesta); saber estar en el mundo sin ponerse uno mismo como centro del mundo (Philippe Meirieu). En definitiva, desde mi punto de vista y teniendo en cuenta la sociedad que nos rodea, contribuir a desarrollar el conjunto de capacidades personales y sociales que nos permitan habitar en el mejor de los mundos posibles. Teniendo en cuenta que la minusvaloración económica y social de la investigación educativa contribuye a aumentar su fragmentación, su irrelevancia, su baja calidad, su baja eficacia y productividad y su baja utilidad (Sancho y Hernández, 1997). Pero, sobre todo, como he señalado, esta situación ayuda a entender la dependencia también continuada de los discursos, las teorías y las propuestas educativas de otras disciplinas y cada vez más del mundo de la empresa, últimamente y de forma especial, de las relacionadas con las tecnologías digitales.

Esta introducción para situar el éxito, que, en los últimos años, ha tenido el discurso de las competencias en las recomendaciones y las políticas educativas. Pero también para entender la dificultad de convertirlo en una práctica educativa. En este artículo, tras situar la irrupción del interés por las competencias y sus múltiples caras, argumentaré, basándome en distintas investigaciones en las que he participado en los últimos años, las posibilidades y dificultades que esta perspectiva ha aportado a la mejora de la educación. 


\section{LA LARGA BÚSQUEDA DE UN APRENDIZAJE CON SENTIDO}

La historia de la educación puede interpretarse como un escenario de lucha o, quizás, como propone Feenberg (1991, p. 15), "una metáfora mejor sería un parlamento de las cosas en el que alternativas civilizatorias se debaten y deciden”. En el mundo occidental, a las concepciones y prácticas sobre la educación subyacen dos visiones que podrían complementarse, pero que suelen contraponerse. La idea de guiar, acompañar, para lograr que aflore y se desarrolle lo mejor de cada individuo -pues, como argumenta Biesta (2016), lo peor también se desarrolla-, que no es considerado como una cabeza hueca, sino como una corporeidad biográfica portadora de experiencias y de un potencial de aprendizaje. Contrasta con la noción de instruir, liderar y conducir el proceso de desarrollo, a menudo sin tener en cuenta al otro (al estudiante) "como un legítimo otro" (Maturana, 1990, p. 24), al que se le suele pedir que deje su identidad en la puerta de la Escuela (Berstein, 1973). La contraposición entre una "mente llena", con todo lo que conlleva de decisiones sobre con qué llenarla, y una "mente bien ordenada" (Morin, 2000), con todo lo que requiere acordar qué entendemos por buen orden, sigue vigente. Y los discurso y las prácticas relacionadas con las competencias no dejan de ser paso más en este largo camino.

Este viaje ha estado marcado por la necesidad de desmarcar el papel de la Escuela como una entidad meramente preservadora y transmisora del pasado (Debray, 2001). De revisar la noción de la enseñanza como recitación, el aprendizaje como repetición y el conocimiento como un recipiente estático (Cuban, 1993). De descolonizar las nociones de la infancia y la juventud (Cannella y Viruru, 2004), que niegan al otro su saber y su capacidad de ser.

Unas visiones impuestas "por el pacto entre la psicología del desarrollo, la medicina, la educación y las agencias gubernamentales" (James, Jenks y Prout, 1998: 17). A estas nociones habría que añadir las que infantilizan la infancia $y$, mediante el consumo y el exceso de protección, la siguen manteniendo en posiciones subordinadas que coartan no solo su capacidad de aprendizaje y acción sino también la asunción progresiva de responsabilidad (Sancho y Hernández, 2014, p. 17).

De cuestionar la función de la evaluación como proceso clasificatorio y represivo orientado a identificar lo que el estudiante no sabe responder en una prueba de papel y lápiz y todas las consecuencias que ello genera (Nichols y Berliner, 2007).

Todas estas y otras cuestiones han jalonado la historia de la innovación educativa desde la irrupción de la Escuela Moderna (Sancho, 2001), pero, sobre todo, desde finales del siglo XIX. En este sentido, en los movimientos de renovación pedagógica vinculados al movimiento progresista (Dewey), la Escuela Nueva (Freinet) y del amplio conjunto de educadores y educadoras que han buscado plantear una educación con sentido, encontramos una intención primigenia de integrar conocimiento, habilidades y actitudes. La base de lo que hoy llamamos competencias. En todo el pensamiento pedagógico innovador hallamos propuestas para promover procesos de enseñanza y aprendizaje centrados en el estudiante, atentos a las problemáticas y desafíos de cada momento histórico, que conciben el conocimiento no como algo que es, sino como algo que deviene y que para que sea significativo ha de ser apropiado por el estudiante con sentido. Con una visión de la evaluación que va más allá de la habilidad para contestar a unas preguntas determinadas, que puede y ha de representar un papel fundamental en el propio proceso de aprender (Earl, Fink, y Stoll, 2007). Apuntando que lo importante no es tanto saber qué, sino cómo, por qué y para qué. 
Sin embargo, estas teorías y prácticas educativas no han tenido un acceso fácil, amplio y profundo en la mayoría de los sistemas escolares, que en palabras de Foucault (1994) se han venido configurando como un potente dispositivo, como una poderosa estructura que componen los discursos, las instituciones, las habilitaciones arquitectónicas, las decisiones reglamentarias, las leyes, las medidas administrativas, los enunciados científicos, las proposiciones filosóficas, morales, filantrópicas [...] el dispositivo siempre está inscrito en un juego de poder, pero también ligado a un límite o a los límites del saber, que le dan nacimiento, pero, ante todo, lo condicionan (p. 229).

Y lo que resulta más inquietante de ese dispositivo es la distancia entre quienes teorizan, investigan y proponen, quienes están al cargo de las políticas educativas, las empresas con considerables intereses económicos en la educación (Rowan, 2002) y las personas inmersas en la práctica.

Por todo lo anterior, mi propuesta en este artículo de situar en un primer momento los discursos sobre las competencias en el campo de la educación -tema sobre el que ya he publicado algunos textos (Sancho, 2011; Sancho y Padilla Petry, 2016). Discutir su incierto camino hacia la práctica y articular algunas conclusiones que nos permitan seguir pensando, aprendiendo y mejorando la educación.

\section{LOS DISCURSOS SOBRE LAS COMPETENCIAS}

Como argumentaba al comienzo del artículo, la complejidad, la multiplicidad de facetas e intereses que convergen en la educación y la falta sistemática de recursos para la investigación educativa, permiten entender la escasez de discursos y propuestas propios y la adopción, poco crítica, de los creados en esferas con mayor relevancia social y sobre todo económica. En este apartado, de forma breve, consideré los más directamente relacionados con las competencias.

\subsection{LOS PROLEGÓMENOS}

En la organización de las instituciones educativas del siglo XX es fácil identificar los elementos de los denominados taylorismo y fordismo. El taylorismo, que como sistema organizativo representaba la quinta esencia de la "administración científica" (Taylor, 1961), se extendió en el contexto del desarrollo industrial de las fábricas, las empresas y la producción, como un instrumento del capital y de la economía. Enseguida, su propuesta de fabricación en cadena, la división de las distintas tareas, los tiempos y espacios del proceso de producción, cuyo fin era aumentar la productividad y evitar el control que el obrero podía tener sobre ellos, obtuvo un éxito indiscutible y se convirtió en un paradigma, en un modelo de eficacia y racionalidad. Al considerarse como aplicable a cualquier organización, la Escuela no escapó a su influencia.

Taylor (1961, p. 30) estaba convencido de que

Cuando se llevan registros exactos de la cantidad de trabajo hecha por cada operario, así como de su eficiencia, y cuando el salario de cada trabajador va aumentándose a medida que la última va mejorando y cuando los que no alcanzan a llegar a un nivel determinado quedan despedidos y en su lugar se da trabajo a un nuevo surtido de operarios cuidadosamente escogidos, cabe suprimir en gran parte tanto la holgazanería natural como el bajo rendimiento sistemático del trabajo.

No lejos de estos principios, el fordismo, que debe su nombre al empresario de la industria del automóvil Henry Ford, -quien implemento, con notable éxito, la línea de ensamble-, a las señaladas características del taylorismo añadió la reducción de costes, la mejora de la circulación de la mercancía, 
el aumento del poder adquisitivo de los asalariados, así como políticas de acuerdo entre obreros organizados (sindicato) y el capitalista.

Estos principios entroncan, en primer lugar, con la visión preponderante de la Escuela del siglo XX como una organización meticulosamente ordenada y con estricta vigilancia, indispensable para la construcción de, en términos de Foucault (1978; 1999), una "sociedad disciplinaria", una "sociedad de control". Una sociedad que se construye a través de una difusa red de dispositivos o aparatos que producen y regulan costumbres, hábitos y prácticas productivas. La obediencia requerida de sus reglas y sus mecanismos de inclusión y/o exclusión se logran mediante instituciones disciplinarias (la prisión, la fábrica, el asilo, el hospital, la universidad, la escuela, etc.) que estructuran lo social a través de lógicas adecuadas a la "razón" de la disciplina. En segundo lugar, a medida que mejora la calidad de vida, con la promesa de acceso a la educación para todas las poblaciones. Y, en tercero, con el discurso de la psicología conductista, que proponía "medir" el rendimiento y la posibilidad de estandarizar los resultados de aprendizaje. Desde una perspectiva que tiende a centrarse en microcompencias aisladas basadas en la idea de lo que alguien hace o debería hacer. Es decir, consistente en una descripción de una acción o un resultado capaz de ser demostrado y evaluado (Norris, 1991). De ahí el enorme impulso que en países como Estados Unidos han tenido las pruebas estandarizadas (Sanders, 1994).

\subsection{LA ECLOSIÓN DEL SIGLO XXI}

En las últimas décadas del siglo XX tuvieron lugar un conjunto de desarrollos científicos, tecnológicos, sociales, políticos y económicos que marcaron el sentido de la entrada al siglo XXI. Una etapa caracterizada por el cambio en los sistemas de producción, el impulso imparable de la globalización, el formidable desarrollo de las tecnologías digitales de la información y la comunicación, el impulso del conservadurismo político y social y el nuevo espíritu del capitalismo (Boltanski y Chiapello, 2002), entre otros. Un periodo que da paso a un sinfín de nuevas acepciones para denominar la sociedad: postcapitalista (Drucker, 1993), postindustrial (Bell,1976); postmoderna (Lyotard, 1984) del conocimiento (Böhme y Stehr, 1986), de la información (Berleur, 1990), de la red (Castells, 2000), del riesgo (Beck, 1992), etc.

\subsubsection{EN EL ÁMBITO EMPRESARIAL}

En este contexto, las formas de organización del trabajo se flexibilizan y aparecen nuevas demandas para los empleados que tienen que adaptarse rápidamente a las exigencias de un mercado cada vez más alejado de la cohesión social y el estado de bienestar (Sennett, 2000 y 2003). Mientras, el fordismo, con su estructura de sindicatos y reivindicaciones salariales y sociales, da paso al toyotismo (Darling-Hammond, 1997). El toyotismo, como forma de organización del trabajo, también debe su nombre a la industria del automóvil, ya que fue desarrollado por la marca japonesa Toyota, para sobreponerse a la crisis del petróleo de comienzos de la década 1970. Los principios de este sistema se basan en: la flexibilidad laboral y la alta rotación en los puestos de trabajo y los roles desempeñados; los estímulos sociales a través del fomento del trabajo en equipo y el liderazgo horizontal; el sistema de producción justo-a-tiempo, que revaloriza la relación entre el tiempo de producción y la circulación de la mercancía, a través de la lógica de menor control de los empleados y reducción de costos de planta. Todo ello desplaza la responsabilidad de la dirección de la empresa al rendimiento de los empleados. Para autores como Rodrigues Ramalho (2011), contribuye de forma significativa al desarrollo de la agenda neoconservadora, al tratase de una nueva cosmovisión en la que, desde la teoría marginal del "capital humano", el individuo es el único responsable de su éxito.

Esta nueva organización del trabajo lleva pareja nuevas aproximaciones a la noción de competencias. Como argumenta Winterton (2009, p. 683), las "medidas" de la competencia comenzaron a utilizarse, en un principio en Estados Unidos, para "ayudar a seleccionar al personal porque las pruebas 
tradicionales de inteligencia cognitiva se consideraban como pobres predictoras del desempeño en el trabajo". La tendencia hacia una perspectiva holística en la gestión de recursos humanos, que comenzó a considerar los atributos psicosociales además de las capacidades cognitivas de los empleados, fue recogida y reforzada por los cambios en la organización del trabajo. El desarrollo imparable de la economía de servicios con su énfasis y exigencia de entrega inmediata -además de la idea de que los productos y servicios no podían mantenerse en stock-, requería, sobre todo, procesos de producción flexibles respaldados por una fuerza de trabajo maleable que pudiera reaccionar ante las cambiantes demandas del mercado. El ascenso estelar de la "competencia" reside precisamente en esta necesidad de flexibilidad, en el sentido que promete una estrategia de pronta respuesta a un mundo cada vez más rápido. Autores como Westera (2001, p. 75) resumen este principio central de la "competencia" al argumentar:

Los empleadores exigen graduados capaces de operar en entornos complejos, es decir, entornos caracterizados por problemas mal definidos, información contradictoria, colaboración informal y procesos abstractos, dinámicos y altamente integrados. El concepto de competencia está fuertemente asociado con la capacidad de dominar situaciones complejas, y se supone que la "competencia" trasciende los niveles de conocimiento y habilidades para explicar cómo se aplican de manera efectiva estos conocimientos y las habilidades.

Teniendo en cuenta que el conocimiento es necesariamente parcial y fragmentado (Lyotard 1984), este enfoque se aleja de las definiciones estrechas y específicas de contenido, apuntando a capacidades más genéricas y abarcadoras, como las estrategias de aprendizaje permanente, que deberían permitir que las personas no quedasen ancladas en lo que aprendieron en su etapa formativa. De ahí el renovado interés y la revalorización de actitudes personales como la disposición y la motivación para aprender. A medida que las rígidas regulaciones tradicionales de los flujos de trabajo concretos se ven desplazadas por la producción ligera basada en equipos autodirigidos, flexibles y con liderazgos distribuidos, otros elementos más allá del conocimiento técnico, como el trabajo en equipo y la resolución colectiva de problemas, son cruciales para un desempeño laboral exitoso. Como argumenta Payne (2000), las habilidades técnicas han sido etiquetadas como actitudes personales porque las presiones de la flexibilidad requerida ponen un nuevo conjunto de demandas holísticas sobre el trabajador individual, ahora compelido a actuar de manera autónoma y a mostrar una alta motivación y liderazgo. El resultado es que las estrechas concepciones funcionales centradas en el contenido de las habilidades laborales, se ponen al lado de capacidades blandas orientadas a la comunicación -tan fundamentales para el cambio hacia una economía de servicios. La competencia consiste en la combinación de las tres dimensiones: las disposiciones actitudinales, las habilidades de orientación prácticas y los conocimientos teóricos necesarios para realizar ciertas tareas (trabajos) de una manera eficiente y excelente.

\subsubsection{EN EL ÁMBITO DE LA EDUCACIÓN}

En el campo de la educación, se multiplican las voces y las iniciativas orientadas a impulsar la transformación de unas instituciones educativas que parecían demasiado ancladas en el pasado y poco al tanto de los retos que planteaba una sociedad en constante evolución. Unas organizaciones que, como señalaba Maria Ferraris (1992, p. 31) en el caso de Italia, carecían de alma, de la chispa y el sentido que debían tener para los millones de personas con el derecho y el deber de pasar largos años de su vida siguiendo su enseñanza.

De este modo nacen propuestas como School of the 21st Century, impulsada por la administración de George H. W. Bush, a comienzos de la década de 1990 en Estados Unidos (Finn-Stevenson, Linkins y Beacom, 1992). O en un contexto social y político diferente, la reforma del sistema educativo promovida por el gobierno socialista en España (BOE, 1990) y puesta en práctica a través de un con- 
siderable cambio curricular, que no solo contemplaba aprendizajes factuales y declarativos, sino también conceptuales, procedimentales, actitudinales y los basados en valores (BOE, 1991a y b). Unas iniciativas respaldadas por organismos internacionales como la UNESCO y las ideas ampliamente reseñadas, mucho menos puestas en práctica, de informes como el de Delors, J. et al. (1996). Este informe consideraba como los puntales de la educación: (a) Aprender a aprender. (b) Aprender a hacer. (c) Aprender a vivir juntos. (d) Aprender a ser. Lo que está en clara sintonía con las ideas, también más estudiadas que practicadas, de los movimientos progresistas y de escuela nueva de finales del XIX y principios del XX. Cada vez se hace más patente, por difícil que parezca cambiar el poderoso dispositivo de la Escuela, la necesidad de ampliar y cuestionar las visiones tradicionales del conocimiento (Gibbons, Limoges, Nowotny, et al., 1994) y considerar el desarrollo de habilidades y actitudes. Lo que subyace a estos movimientos es el reto que supone educar a estudiantes del siglo XXI, con docentes del siglo XX y escuelas del XIX (Sancho y Brain, 2013), considerando la dificultad de cambiar el imaginario pedagógico de la mayoría de los implicados en los sistemas educativos (Sawyer, 2008).

En este contexto, son los organismos internacionales quienes promueven y desarrollan los discursos vinculados a la necesidad de dar respuesta a los desafíos educativos y formativos de un mundo cambiante, a través de la enseñanza y el desarrollo de competencias.

Según Ananiadou y Claro (2009), la perspectiva de la OCDE sobre las nuevas competencias y habilidades ha sido elaborada a través de dos importantes iniciativas: el Programa de Definición y Selección de Competencias (DeSeCo) y Programa Internacional de Evaluación del Estudiante (PISA, por sus siglas en inglés). En el proyecto DeCeSo de la OCDE (2002), parece claro que la noción de competencia implica varias dimensiones - no demasiado alejadas de la propuesta del informe Delors- a partir de la capacidad de (1) de utilizar una amplia variedad de herramientas, (2) interactuar con otros en grupos heterogéneos y (3) asumir la responsabilidad de la propia vida y actuar de manera autónoma. En el centro de las competencias clave, el documento sugiere reflexividad. Siendo coherente con el diagnóstico inicial de un mundo globalizado, la capacidad de manejar las diferencias y de tratar con las contradicciones que definen las situaciones no estándar y complejas parece evidente. "El mundo actual, diverso y complejo, exige que no nos precipitemos necesariamente a una sola respuesta, a una solución o bien, sino que manejemos las tensiones [...] integrando objetivos aparentemente contradictorios o incompatibles como aspectos de una misma realidad" (OCDE 2002, p. 9).

El impacto educativo de propuesta de la OCDE, se basa en que los resultados del Proyecto DeSeCo constituyeron los fundamentos teóricos de PISA, que como es sabido trata de supervisar hasta qué punto los estudiantes, al final de la enseñanza obligatoria, han adquirido el conocimiento y las habilidades consideradas necesarias para participar activamente en la sociedad. "Se centra en la capacidad de los jóvenes para utilizar su conocimiento y habilidades para enfrentar los desafíos reales de la vida, más que hasta qué punto han dominado un determinado currículo escolar" (Ananiadou y Claro, 2009, p. 7). No entraremos aquí en las múltiples y críticas articuladas en torno a las pruebas PISA (Bautier y Rayou, 2007; Carabaña, 2015, entre otros), pero sí señalaremos brevemente la "profusión" de definiciones y aproximaciones a las competencias que abordaremos más adelante. Una situación difícil de seguir y entender por parte de un profesorado que se enfrenta cada día con un trabajo de alta complejidad.

El Proyecto DiSeCo realiza una distinción importante en relación a los términos "habilidades" y "competencias" y como se relacionan:

Una competencia es más que solo conocimiento o habilidades. Implica la habilidad de responder a demandas complejas, dibujando o movilizando recursos psicosociales (incluyendo habilidades y actitudes) en un contexto particular. Por ejemplo, la habilidad de comunicarse de forma efectiva es una competencia que puede basarse en el conocimiento individual de la lengua, habilidades prácticas de TI y actitudes 
hacia aquellos con los que uno se está comunicando (Rychen y Salganik, 2003, citados por Ananiadou y Claro, 2009, p. 8).

Por otro lado, teniendo en cuenta la profunda relación de las competencias con el mundo del trabajo, la considerable ambigüedad encontrada en la bibliografía se refiere a la confluencia entre la competencia como resultados (normas profesionales que describen lo que la gente necesita hacer en el trabajo), como tareas que las personas deben realizar (lo que han de hacer ahora), y como rasgos o características personales (cómo son las personas) (Mansfield 2004). Además, en los estudios sobre competencias asociadas a la educación, surge una contradicción ligeramente diferente e incluso más profunda. Cuando la competencia comienza a ser considerada como un estándar educativo que debe guiar la evaluación, su orientación genérica se convierte en su principal desventaja. Para Westera (2001, p. 86), "las competencias están asociadas a comportamientos exitosos en situaciones no estandarizadas", mientras que tal definición está en desacuerdo con "el uso de competencias como estándares educativos". Es decir, la misma fuerza y necesidad de un enfoque de aprendizaje basado en la "competencia" y el currículo, se convierten a la vez en su principal problema, ya que enfrentarse a situaciones complejas (mal definidas, no estandarizadas) no puede basarse y no se basa en soluciones preconfeccionadas que puedan ser fácilmente operacionalizadas y aplicadas en entornos educativos de gran escala.

En este contexto, en 2006, la Comisión Europea definió las 8 competencias clave (entendidas como una combinación de conocimientos, habilidades y actitudes) consideradas como necesarias para la realización personal, la ciudadanía activa, la cohesión social y la empleabilidad en una sociedad del conocimiento.

1. Comunicación en la lengua materna.

2. Comunicación en lenguas extranjeras.

3. Competencia matemática y competencias básicas en ciencia y tecnología.

4. Competencia digital.

5. Aprender a aprender.

6. Competencias sociales y cívicas.

7. Sentido de la iniciativa y espíritu de empresa.

8. Conciencia y expresión culturales.

Aquí se puede observar la dificultad que supone referirse a la competencia en general, cuando a menudo el enfoque más holístico no alcanza niveles suficientes de concreción. Esto se puede observar en relación con lo que nos interesa más en este artículo, a saber, las competencias digital y sociales y cívicas.

La definición de la CE estipula que

La competencia digital entraña el uso seguro y crítico de las tecnologías de la sociedad de la información (TSI) para el trabajo, el ocio y la comunicación. Se sustenta en las competencias básicas en materia de TIC: el uso de ordenadores para obtener, evaluar, almacenar, producir, presentar e intercambiar información, y comunicarse y participar en redes de colaboración a través de Internet. (EC 2007, p. 15).

A la vez que estipula los conocimientos, capacidades y actitudes que considera esenciales en relación a esta competencia. Lo mismo sucede en el caso de las competencias sociales y cívicas.

Para los miembros de la Comisión.

Estas competencias incluyen las personales, interpersonales e interculturales y recogen todas las formas de comportamiento que preparan a las personas para participar de una manera eficaz y constructiva en la vida social y profesional, especial- 
mente en sociedades cada vez más diversificadas, y, en su caso, para resolver conflictos. La competencia cívica prepara a las personas para participar plenamente en la vida cívica gracias al conocimiento de conceptos y estructuras sociales y políticas, y al compromiso de participación activa y democrática (EC 2007, p. 16).

Pero, como es sabido, ni la OCDE, ni la Comisión Europea pueden intervenir en la organización de los sistemas educativos de los distintos países. De este modo, sus propuestas, incluidas las pruebas PISA, se hubieran quedado en el papel si los gobiernos de los distintos países no hubiesen decidido adoptarlas. Algo que sucedió en España en 2006, al promulgarse el Real Decreto 1631/2006, de 29 de diciembre, por el que se establecen las enseñanzas mínimas correspondientes a la Educación Secundaria Obligatoria (BOE, 2007a). En este documento, las 8 competencias básicas, que se supone toda persona ha de haber podido desarrollar o adquirir mínimamente al acabar la escolaridad obligatoria son:

1. Competencia en comunicación lingüística.

2. Competencia matemática.

3. Competencia en el conocimiento y la interacción con el mundo físico.

4. Tratamiento de la información y competencia digital.

5. Competencia social y ciudadana.

6. Competencia cultural y artística.

7. Competencia para aprender a aprender.

8. Autonomía e iniciativa personal.

Según este decreto, mediante la inclusión de las competencias básicas en el currículo, que algunos comienzan a ver demasiado abultado de contenidos, se intenta integrar los aprendizajes de las diferentes áreas o materias, con los informales y no formales; permitir que todos los estudiantes pongan en relación sus aprendizajes con distintos tipos de contenidos y utilizarlos de manera efectiva cuando les resulten necesarios en diferentes situaciones y contextos; y orientar la enseñanza, al permitir identificar los contenidos y los criterios de evaluación que tienen carácter imprescindible y, en general, inspirar las distintas decisiones relativas al proceso de enseñanza y de aprendizaje (BOE, 2007a, p. 285).

El decreto, que es una norma jurídica con rango de ley de obligado cumplimiento al ser convalidada por el poder legislativo, sigue especificando un conjunto de prescripciones:

El trabajo en las áreas y materias del currículo para contribuir al desarrollo de las competencias básicas debe complementarse con diversas medidas organizativas y funcionales, imprescindibles para su desarrollo. Así, la organización y el funcionamiento de los centros y las aulas, la participación del alumnado, las normas de régimen interno, el uso de determinadas metodologías y recursos didácticos, o la concepción, organización y funcionamiento de la biblioteca escolar, entre otros aspectos, pueden favorecer o dificultar el desarrollo de competencias asociadas a la comunicación, el análisis del entorno físico, la creación, la convivencia y la ciudadanía, o la alfabetización digital. Igualmente, la acción tutorial permanente puede contribuir de modo determinante a la adquisición de competencias relacionadas con la regulación de los aprendizajes, el desarrollo emocional o las habilidades sociales. Por último, la planificación de las actividades complementarias y extraescolares puede reforzar el desarrollo del conjunto de las competencias básicas (Ibid., p. 285).

A la vez que especifica qué entiende y qué implica, en nuestro caso, el desarrollo de la competencia de tratamiento de la información y competencia digital. 
Esta competencia consiste en disponer de habilidades para buscar, obtener, procesar y comunicar información, y para transformarla en conocimiento [...] implica ser una persona autónoma, eficaz, responsable, crítica y reflexiva al seleccionar, tratar y utilizar la información y sus fuentes, así como las distintas herramientas tecnológicas; también tener una actitud crítica y reflexiva en la valoración de la información disponible, contrastándola cuando es necesario, y respetar las normas de conducta acordadas socialmente para regular el uso de la información y sus fuentes en los distintos soportes (Ibid., p. 285).

Y de competencia social y ciudadana.

Esta competencia hace posible comprender la realidad social en que se vive, cooperar, convivir y ejercer la ciudadanía democrática en una sociedad plural, asî como comprometerse a contribuir a su mejora. En ella están integrados conocimientos diversos y habilidades complejas que permiten participar, tomar decisiones, elegir cómo comportarse en determinadas situaciones y responsabilizarse de las elecciones y decisiones adoptadas [...] supone comprender la realidad social en que se vive, afrontar la convivencia y los conflictos empleando el juicio ético basado en los valores y prácticas democráticas, y ejercer la ciudadanía, actuando con criterio propio, contribuyendo a la construcción de la paz y la democracia, y manteniendo una actitud constructiva, solidaria y responsable ante el cumplimiento de los derechos y obligaciones cívicas Ibid., p. 289).

Llegados a este punto la gran pregunta es ¿cómo cumple en la Escuela este decreto ley de obligado cumplimiento?

\section{EL LARGO CAMINO DEL PAPEL A LA PRÁCTICA EDUCATIVA}

En los últimos años, en nuestro grupo de investigación hemos realizado distintos estudios que nos han permitido vislumbrar las posibilidades y dificultades de llevar a la práctica una enseñanza capaz de traspasar los rígidos límites de las concepciones tradicionales sobre el conocimiento, la enseñanza y el aprendizaje.

En el Proyecto europeo "School+ More than a Platform to Build the School of Tomorrow" (Unión Europea. IST Programme. V Framework Porgramme. 2000-IST-25162), llevado a cabo en 25 institutos de cinco países europeos, pusimos a los estudiantes en el centro del proceso de un aprendizaje orientado a la comprensión y la dotación de sentido. Para ello desarrollamos una plataforma digital de trabajo colaborativo, con el fin de promover el uso educativo de las TIC junto a perspectivas contemporáneas sobre la enseñanza y el aprendizaje basadas en proyectos de indagación (Hernández y Ventura, 2008). A su vez, propusimos favorecer la reorganización del entorno escolar para promover la integración y adaptación de las TIC a las necesidades emergentes de los centros y su tarea de educar individuos con predisposición para seguir aprendiendo a lo largo de la vida.

El profesorado de los institutos, junto con los investigadores de la universidad y una empresa, participaron de forma colaborativa y horizontal en todo el proceso de toma de decisiones e implementación de las mismas, lo que propició la consecución de los objetivos planteados y una considerable transformación de las situaciones de enseñanza y aprendizaje. El proyecto también nos permitió vislumbrar que, los problemas de la implementación de nuevas perspectivas de enseñanza y aprendizaje incorporando las TIC se situaban en: 
- Las sobrecargadas especificaciones y los niveles de los currículos.

- Las restricciones que provienen de la propia Administración.

- Los esquemas organizativos de la enseñanza (lecciones de 45-50 minutos).

- La organización del espacio -acceso a los ordenadores, número de estudiantes por clase...

- Los sistemas de formación permanente del profesorado que impiden el cambio educativo.

- El contenido disciplinar de los currículos que dificultan las propuestas transdisciplinares y el aprendizaje basado en problemas.

- Las restricciones en la organización del espacio y el tiempo.

- La falta de motivación por parte del profesorado para introducir nuevos métodos.

- La poca autonomía que gozan el profesorado y el alumnado (Sancho, 2006, p. 32).

El desarrollo de los proyectos a los que me refiero a continuación, nos ha llevado a analizar un gran número de documentos oficiales. En este trabajo, en relación a las competencias, hemos podido constatar la distancia entre las recomendaciones de los organismos internaciones, las prescripciones de las políticas educativas y las prácticas de los centros. La falta de una definición operativa de lo que significa poner en práctica en una enseñanza de tradición disciplinar una basada en competencias, ha llevado a las consejerías de las distintas comunicadas autónomas a publicar un conjunto de documentos que contienen una profusión de niveles de concreción, desarrollo y propuestas más o menos prácticos (Departament d'Educació, 2010; Departamento de Educación, Universidades e Investigación, $\mathrm{s} / \mathrm{f}$; Departament d'Ensenyament, 2017; http://www.juntadeandalucia.es/educacion/agaeve/profesorado-primaria-evaluacion.html, entre muchos otros). Unas publicaciones que difícilmente llegan a un profesorado no suele disponer de tiempo y espacios de formación para analizar lo que supone esta perspectiva de enseñanza y diseñar las condiciones para llevarla a la práctica.

Una situación que revela que, como sostienen Grugulis, Warhurst y Keep (2004, p. 8), el atractivo retórico de las competencias genéricas defendidas por los gobiernos tiene que contrastarse con las “intratables dificultades de implementación”. Para van der Klink y Boon (2003), el mayor problema es que, a pesar de la comprensión del valor limitado de las definiciones funcionales, su comprensión más holística se basa precisamente en ser un concepto demasiado difuso. Además, aunque la definición de competencia en el ámbito educativo apunta claramente a características transversales más genéricas, Fischer, Bullock, Rotenberg y Raya (1993) insisten en que la competencia de las personas es siempre una competencia en contexto. Lo que se entiende por competencia depende del lugar concreto en el que las personas operan. Sin embargo, esto implica que la definición de competencia se vuelve tan variada como los diferentes contextos sociales, culturales y laborales.

Todas las instituciones con las que hemos llevado a cabo las investigaciones, y digo con, porque no investigamos sobre las personas y las organizaciones sino con ellas (Hernández, 2011; Nind, 2014), incorporan en sus Planes de Centro y Diseños Curriculares, referencias específicas al desarrollo de las competencias propuestas en los documentos oficiales. Los elementos que configuran las competencias sociales y cívicas, se suelen encuentrar contempladas en la organización del centro y como garantes de la convivencia. Las formas de evaluación de estas competencias, en general difusas, se sitúan tanto en el comportamiento visible del alumnado en el nivel general, como en el de los distintos espacios de enseñanza. El tema del tratamiento de la información y la competencia digital, conlleva otras problemáticas.

En proyecto "Políticas y prácticas en torno a las TIC en la enseñanza obligatoria: Implicaciones para la innovación y la mejora" (MICINN. SEJ2007-67562), estudiamos en profundidad, cómo las 
políticas educativas en torno a las TIC transitaban hasta dos institutos y dos escuelas primarias. La temática del proyecto llevó a centrar nuestra atención en el tratamiento de la información y la competencia digital que, por supuesto, estaba presente en los documentos organizativos y curriculares de las cuatro instituciones. Mientras que en la práctica educativa se encontraba sobre todo en asignaturas dedicadas a enseñar y aprender sobre las TIC, o en aquellas en las que las TIC se utilizaban, para enseñar (mostrar) y aprender (ejercitar). Es decir, la parte más instrumental de la esta competencia. En mucha menor medida encontramos momentos, siempre relacionados con rupturas del currículo tradicional -como el "proyecto de síntesis de $3^{\circ}$ de ESO, o el proyecto de investigación de $4^{\circ}$ (Sancho, Padilla Petry, Domingo, Müller y Giro, X. (2012) o la integración de varias disciplinas (Ornellas, Moltó, Guitert, y Romeu, 2012).

Este proyecto nos permitió vislumbrar lo que consideramos como cuatro puntales para la mejora de la educación mediada por las TIC, como base para el desarrollo del tratamiento de la información y la competencia digital (Sancho, Giró, Ornellas, Sánchez, Fraga, Guitert y Alonso, 2012). El primero se refiere a la necesidad de no descuidar la (in)estable relación de las políticas y las prácticas. Una buena parte del profesorado implicado mostraba un cierto, o gran, cansancio por los cambios constantes de las políticas educativas, sin contar para nada con ellos. Daba la impresión, por lo dicho y no dicho, que contaban con que los políticos y las políticas pasan y los centros se quedan con los problemas no resueltos. De aquí el título del libro que da cuanta del conjunto de la investigación: La fugacidad de las políticas, la inercia de las prácticas (Sancho y Alonso, 2012). El segundo tiene que ver con la infraestructura y la obsolescencia, debido a la imposibilidad de los centros de mantener al día unos equipos y aplicaciones que crecen a velocidad exponencial. El tercero con la complejidad de las dimensiones y las condiciones del uso de las TIC para la innovación y la mejora, que ni la Administración, ni los centros parecen en condiciones de abordar. El cuarto, y no menos importante, con la formación del profesorado que, caso de que exista, se tiende a centrar más en la tecnología que en la pedagogía.

El proyecto "Identidoc - La construcción de la identidad docente del profesorado de educación primaria en la formación inicial y los primeros años de trabajo" (MICINN. EDU2010-20852-C02-01), en el que colaboraron más de 50 maestros y maestras de distintas comunidades, nos permitió evidenciar un importante problema. Estos docentes, que en 2012 se encontraban entre su primer y quinto año de vida profesional, es decir, que, en principio, seguirían realizando su trabajo en los próximos treinta años, además de encontrarse en una situación laboral de gran incertidumbre, reconocían no contar con formación para afrontar los retos de la sociedad digital (Sancho y Hernández, 2012). La suya había sido una formación disciplinar y compartimentalizada, basada en las asignaturas tradicionales y, en general, orientada a la transmisión de contenido. Sin que la perspectiva competencial del conocimiento hubiese encontrado su lugar en su experiencia formativa. Los currículos docentes desarrollados a partir de la conversión de la diplomatura en grado, de la mano de la instauración del Espacio Europeo de Educación Superior, tampoco parecen haber mejorado esta situación. De hecho, las competencias que según ORDEN ECI/3857/2007, de 27 de diciembre, por la que se establecen los requisitos para la verificación de los títulos universitarios oficiales que habiliten para el ejercicio de la profesión de Maestro en Educación Primaria (BOE, 2007b), se refieren a la capacidad de enseñar las distintas asignaturas del currículo de primaria. Por otro lado, aunque las distintas universidades recogen un listado de competencias a desarrollar por los estudiantes de magisterio, los currículos siguen siendo extremadamente disciplinares y fragmentados (Sancho-Gil, Sánchez-Valero y Domingo-Coscollola, 2017) y todo lo referido a la competencia digital sigue siendo un eco (Sancho y Brain, 2013).

En proyecto "Vivir y aprender con nuevos alfabetismos dentro y fuera de la escuela secundaria: aportaciones para reducir el abandono, la exclusión y la desafección escolar de los jóvenes. (MICINN. 
EDU2011-24122), treinta y nueve jóvenes de cinco institutos de Cataluña, acompañados por 14 investigares y profesores, realizaron 5 etnografías sobre cómo, con quién y con qué se expresaban, aprendían y comunicaban dentro y fuera de los centros (Hernández-Hernández, 2017). Este estudio mostró cómo los estudiantes son más capaces que los docentes de conectar lo que sucede dentro con lo que pasa fuera. Y cómo los jóvenes desarrollan y se benefician más del tratamiento de la información y la competencia digital fuera de los institutos. También evidenció una falta de la educación crítica sobre las implicaciones personales y sociales del desarrollo y el uso de las tecnologías digitales.

Finalmente, en el proyecto europeo "DIYLab Do it yourself in Education: Expanding Digital Competence to foster Student Agency and Collaborative Learning - DIYLab" (European Commision). Education, Audiovisual and Culture Executive Agency. 543177-LLP-1-2013-1-ES-KA3MP, tres escuelas primarias y secundarias y dos universidades de España, Finlandia y Chequia, nos propusimos explorar el desafío de introducir la cultura "hazlo por ti mismo", a lo que añadimos en colaboración, para profundizar en la competencia digital. Un proceso riguroso de investigación en la acción participativa (Reason y Bradbury, 2001; Sancho-Gil y Rivera-Vargas, 2016), nos permitió trabajar desde la noción de liderazgo para el aprendizaje (Domingo-Coscollola, Arrazola-Carballo y SanchoGil, 2016). Una posición que posibilitó involucrar a todos los estamentos de la comunidad educativa, identificar espacios institucionales e informales en los que anclar esta perspectiva de aprendizaje, llevar a cabo proyectos de indagación con un elevado nivel de autonomía y de desarrollo de la competencia digital por parte del alumnado, y mostrar los avances de los estudiantes a través de la elaboración de objetos visuales digitales compartidos a través de una plataforma digital: http://hub.diylab.eu/.

También llevó al desarrollo de rúbricas de autovaluación, coevalaución y heteroevaluación de la competencia digital. Pero lo más importante, para los miembros de la Escola Virolai de Barcelona, una de las instituciones participantes, fue que el proyecto

"se ha establecido en la comunidad educativa y la cultura DIY se ha convertido en parte de nuestro enfoque educativo: una escuela para aprender, una escuela que aprende". Esto nos ha llevado a repensar nuestros roles docentes para fomentar el aprendizaje de los estudiantes a través de una autonomía relevante y significativa y una creatividad colectiva, acercándonos a situaciones reales de manera transdisciplinaria y colaborativa, teniendo en cuenta las habilidades y el conocimiento de todos los participantes. Esto ha promovido la implementación de cambios curriculares, metodológicos y organizativos, especialmente en el sistema de evaluación, basando todo en la reflexión individual y compartida sobre el proceso de aprender qué, cómo, cuándo, dónde, por qué, con quién y para qué (DIYLab, 2016, p. 19).

Esto ha podido ser así, porque no se trata de una idea impuesta, sino de un proceso discutido, analizado e impulsado por todos los participantes. Porque como argumentaba Lawrence Stenhouse, somos malos implementadores de las ideas de otros.

\section{Conclusiones. PARA SEguir PENSANDO}

Este artículo pone de manifiesto una serie de temáticas relacionadas con la educación actual en relación al tema de las competencias. La primera se refiere a la persistente y cada vez más pronunciada dependencia de la educación de los discursos, necesidades e intereses del mundo empresarial. Los análisis sobre los desafíos de la educación en el mundo contemporáneo vienen cada vez más marcados 
por una concepción neoconservadora de la economía y el trabajo y el tema de las competencias parece el penúltimo paso de este proceso.

La segunda, estrechamente relacionada con la anterior, tiene que ver con el papel de los organismos internacionales a la hora de marcar las agendas educativas. Unas agendas que los distintos gobiernos adoptan, por razones de distinto tipo, sin contar con los directamente implicados en su ejecución y sin garantizar las condiciones necesarias para poder ponerlas realmente en la práctica.

La tercera está directamente conectada con el conjunto de dimensiones y dilemas que acompañan al mandato de introducir en tradiciones escolares fuertemente disciplinares, perspectivas basadas en las competencias. Y hacerlo, además, sin proporcionar los medios necesarios para garantizar la formación del profesorado y los recursos necesarios para quienes las han de llevar a la práctica.

La cuarta, parte de la consideración de que la introducción de una perspectiva de por sí controvertida y multifacética, como la de las competencias, sin contar con todos o con la mayoría de los participantes en el análisis y discusión de qué supone y en la planificación de los posibles escenarios para ponerla en práctica, parece, como evidencia este texto, bastante abocada al fracaso.

El considerable saber disponible sobre el cambio educativo (Fullan, 2007a; 2007b; Hargreaves y Shirley, 2012; Sarason, 2003) nos advierte de la dificultad de introducir transformaciones significativas en las instituciones de enseñanza sin contar con quienes las han de llevar a cabo. También del peligro de proponer un cambio tras otros para que todo continúe igual.

Finalmente, el panorama narrado en este texto, nos llama a todos los educadores, pero sobre todo a los que nos dedicamos a la investigación y la formación, a poner una atención especial en cómo se generan los pensamientos neocoloniales que emergen de las grandes corporaciones y qué consecuencias tienen para la educación de la mayoría. Porque el desafío más importante, para mí, lo plantea la necesidad de contribuir a crear discursos y prácticas educativas inclusivas, en su sentido más amplio, incluso para el maltratado medio ambiente.

\section{AGRADECIMIENTOS}

Grupo de investigación ESBRINA-Subjetividades, visualidades y entornos educativos contemporáneos (2014SGR 00632).

Red de excelencia REUNI+D- Red Universitaria de Investigación e Innovación Educativa. Cambios Sociales y Retos para la Educación en la Era Digital. EDU2015-68718-REDT.

\section{BIBLIOGRAFÍA}

Álvarez, P. (2016, junio 22). El gasto educativo cae 8.920 millones y baja a niveles de hace 25 años. El País. Recuperado https://politica.elpais.com/politica/2016/06/22/actualidad/1466585749_283595.html

Ananiadou, K. y Claro, M. (2009). 21st Century Skills and Competences for New Millennium Learners in OECD Countries. OECD Education Working Papers, No. 41. Paris: OECD Publishing. http://dx.doi.org/10.1787/218525261154

Banks, J. A., Au, K. H., Ball, A. F., Bell, P., Gordon, E. W., Gutiérrez, K.,... Zhou, M. (2007). Learning in and out of school in diverse environments. Life-long, life-wide, life-deep. The LIFE Center for Multicultural Education. Recuperado de https://goo.gl/Cu2R6J

Bautier, E. y Rayou, R. (2007). What PISA really evaluates: literacy or students' universes of reference? Journal of Educational Change, 8(4), 359-364. doi: http://dx.doi.org/10.1007/s10833-0079043-9. 
Beck, U. (1992). Risk Society. Towards a New Modernity. London; Thousand Oaks, Calif.: Sage Publications.

Bell, D. (1976). El advenimiento de la sociedad post-industrial: Un intento de prognosis social. Madrid: Alianza Editorial.

Berleur, J. (Ed.) (1990). The Information society: evolving landscapes. New York: Springer-Verlag.

Berstein, B. (1973). Education cannot compensate for society. En J. Raynor y J. Harden (Eds.), Equality and City Schools. Readings in Urban Education Vol. 2. London: Routledge and Kegan Paul.

Biesta, G. J. (2016). Good Education in an Age of Measurement: Ethics, Politics, Democracy. New York: Routledge.

BOE (1990). Ley orgánica 1/1990, de 3 de octubre de 1990, de Ordenación General del Sistema Educativo. BOE, 238, 28927-28942.

BOE (1991b). Real Decreto 1006/1991, de 14 de junio, por el que se establecen las enseñanzas mínimas correspondientes a la Educación Primaria. BOE, 152. Recuperado de https://www.boe.es/boe/dias/1991/06/26/

BOE (1991b). Real Decreto 1007/1991, de 14 de junio, por el que se establecen las enseñanzas mínimas correspondientes a la Educación Secundaria Obligatoria. BOE, 152. Recuperado de https://www.boe.es/boe/dias/1991/06/26/

BOE (2007a). REAL DECRETO 1631/2006, de 29 de diciembre, por el que se establecen las enseñanzas mínimas correspondientes a la Educación Secundaria Obligatoria. BOE, 5, 677 - 773. Recuperado de https://www.boe.es/boe/dias/2007/01/05/pdfs/A00677-00773.pdf

BOE (2007b). ORDEN ECI/3857/2007, de 27 de diciembre.” BOE 312: 53747- 53750. Recuperado de https://www.boe.es/boe/dias/2007/12/29/pdfs/A53747-53750.pdf

Böhme, G. y Stehr, N. (1986). The Knowledge society: the growing impact of scientific knowledge on social relations. Dordrecht; Boston: D. Reidel Pub. Co.

Boltanski, L. y Chiapello, È. (2002). El nuevo espiritu del capitalismo. Madrid: Akal.

Cannella, G. y Viruru, R. (2004). Childhood and Postcolonization. New York \& London: Routledge.

Carabaña, J. (2015). La inutilidad de PISA para las escuelas. Madrid: Los Libros de la Catarata.

Castells, M. (2000). The rise of the network society. Oxford \& Malden, MA.: Blackwell Publishers.

Cuban, L. (1993). How teachers tanght: constancy and change in American classrooms, 1890-1990. New York: Teachers College Press.

Darling-Hammond, L. (1997). The right to learn. San Francisco: Jossey-Bass Pu.

Debray, R. (2001). Introducción a la mediología. Barcelona: Paidós.

Delors, J. et al. (1996). La educación encierra un tesoro. Madrid: Santillana.

Departament d'Educació (2010). Currículum d'Educació Secundària Obligatòria. Barcelona: Generalitat de Catalunya. Departament d'Educació. Recuperado de: http://xtec.gencat.cat/.content/alfresco/d/d/workspace/SpacesStore/0023/fe124c3b-263244ff-ac26-dfe3f8c14b45/curriculum eso.pdf

Departamento de Educación, Universidades e Investigación (s/f). Las competencias básicas en el Sistema Educativo de la C.A.P.V. Gobierno Vasco. Recuperado de http://www.hezkuntza.eigv.euskadi.eus/contenidos/informacion/dig publicaciones innovacio n/es curricul/adjuntos/14 curriculum competencias 300/300002c Pub BN Competencias Basicas c.pdf

Departament d'Ensenyament (2017). Programar per competències a l'educació primària. Una eina per a la reflexió pedagògica $i$ la presa de decisions dels equips docents Generalitat de Catalunya. Departament d'Ensenyament. 
http://ensenyament.gencat.cat/web/.content/home/departament/publicacions/colleccion s/competencies-basiques/primaria/programar-educacio-primaria.pdf

DIYLab (2016). Final Report. Public Part. Recuperado de http://diylab.eu/docs/DIYLab public Final report llp en2016 FF.pdf

Domingo-Coscollola, M., Arrazola-Carballo, J., and Sancho-Gil, J.M. (2016). Do It Yourself in education: Leadership for learning across physical and virtual borders. International Journal of Educational Leadership and Management, 4(1), 5-29. doi:10.17583/ijelm.2016.02

Drucker, Peter (1993). Post-capitalist society. New York, NY.: Harper Business.

Earl, L., Fink, D. y Stoll, L. (2007). Sobre el aprender y el tiempo que requiere. Barcelona: Octaedro.

European Communities (EC) (2007). RECOMEND ACIÓN DEL PARLAMENTO EUROPEO Y

DEL CONSEJO de 18 de diciembre de 2006 sobre las competencias clave para el aprendizaje permanente. Luxembourg: Office for Official Publications of the European Communities. Recuperado de http://eur-lex.europa.eu/legalcontent/ES/TXT/PDF/?uri=CELEX:32006H0962\&from=EN

Feenberg, A. (1991). The Critical Theory of Technology. New York: Oxford University Press.

Ferraris, M. (1992). Information Technology in Italian School System: some problems and perspectives. En J. M. Sancho (coord.), European Conference about Information Technology in Education: a critical insight. Lectures (pp. 31-51). Barcelona: Universidad de Barcelona.

Finn-Stevenson, M., Linkins, K. y Beacom, E. (1992). The school of the 21st century: Creating opportunities for school-based child care. Child Youth Care Forum, 21(5), 335-345. https://doi.org/10.1007/BF00757383

Fischer, K.W., Bullock, D.H., Rotenberg, E.J. y Raya, P. (1993). The dynamics of competence: how context contributes directly to skill. En R.H. Wozniak y K. W. Fischer (eds.), The Jean Piaget symposium series. Development in context: Acting and thinking in specific environments (pp. 93-117). Hillsdale, NJ: Lawrence Erlbaum Associates.

Foucault, M. (1978). Microfisica del poder. Madrid. La Piqueta.

Foucault, M. (1999). Vigilar y castigar. Barcelona: Círculo de Lectores.

Foucault (1994). Dits et ecrits. París: Gallimard.

Fullan, M. (2007a). Liderar en una cultura de cambio. Barcelona: Octaedro.

Fullan, M. (2007b). Los nuevos significados del cambio en la educación. Barcelona: Octaedro.

Grugulis, I, Warhurst, C y Keep, E. (2004). 'What's happening to skill?'. En C. Warhurst, I. Grugulis y E. Keep (eds.), The Skills that matter. (pp. 1-18). New York: Palgrave Macmillan.

Gibbons, M., Limoges, C., Nowotny, H., Schwartzman, S., Scott, P. \& Trow, M. (1994). The New Production of Knowledge: The Dynamics of Science and Research in Contemporary Societies. London: Sage Publications.

Hargreaves, A. y Shirley, D. (2012). La cuarta vía. El prometedor futuro del cambio educativo. Barcelona: Octaedro.

Hernández, F. (coord.) (2011). Investigar con los jóvenes: cuestiones temáticas, metodológicas, éticas y educativas. Barcelona: Depósito digital de la UB. http://diposit.ub.edu/dspace/handle/2445/17362

Hernández-Hernández (Coord.) (2017). ¡Y luego dicen que la escuela pública no funciona! Investigar con los jóvenes sobre cómo transitan y aprenden dentro y fuera de los centros de Secundaria. Barcelona: Octaedro.

Hernández, F. y Ventura, M. (2008). La organización del curriculum por proyectos de trabajo. El conocimiento es un calidoscopio. Barcelona: Octaedro. (Primera edición 1992). 
Husén, T. (1988). Nuevo análisis de la sociedad del aprendizaje. Barcelona: Paidós.

Lyotard, J. F. (1984). La condición postmoderna: informe sobre el saber. Madrid: Cátedra.

Mansfield, B. (2004). Competence in transition. Journal of European Industrial Training, 28, (2/3/4), 296309.

Maturana, H. (1990). Emociones y lenguaje en educación y política. Santiago: Ediciones Dolmen.

Morin, E. (2000). La mente bien ordenada. Barcelona: Seix Barral.

Nichols, S. L \& Berliner, D. (2007). Collateral damage: how high-stakes testing corrupts America's schools. Cambridge, MA: Harvard Education Press.

Nind, Melanie (2014). What is inclusive research? London: Bloomsbury.

Norris, N. (1991) The trouble with competence, Cambridge Journal of Education, 21(3), 331-341.

OECD. 2002. The Definition and Selection of Key Competencies. Executive Summary. Paris: OECD. Recuperado de http://www.oecd.org/dataoecd/47/61/35070367.pdf

Ornellas, A., Moltó, O., Guitert, M. y Romeu, T. (2012). El Instituto Salvador Espriu. Acercar la enseñanza al mundo actual. En J. M. Sancho y C. Alonso (Coord.), Op. cit. (pp. 35-60).

Pardos, P. (2017, marzo 23). España doblará su gasto en Defensa en siete años. El Mundo. Recuperado de http://www.elmundo.es/espana/2017/03/23/58d442c5268e3e0c108b464e.html

Payne, J. (2000). The unbearable lightness of skill: the changing meaning of skill in UK policy discourses and some implications for education and training. Journal of Education Policy, 15(3), 353369.

Reason, P. y Bradbury, H. (2001). Handbook of Action Research: Participative Inquiry and Practice. London: Sage.

Rodrigues Ramalho, R. (2011). Gestión toyotista: sindicalización como factor de producción y la cooptación intelectual del trabajador. IX Jornadas de Sociología. Facultad de Ciencias Sociales. Buenos Aires: Universidad de Buenos Aires. Recuperado de http://cdsa.aacademica.org/000034/424.pdf

Rowan, B. (2002). The Ecology of School Improvement: Notes on the School Improvement Industry in the United States. Journal of Educational Change, 3(3-4), 283-314.

Sanders, J. R. (Chair) (1994). The program evaluation standards: how to assess evaluations of educational programs. Thousand Oaks [Calif.]: Sage Publications.

Sancho, J. M. (2001). Repensando el significado y metas de la educación en la sociedad de la información. El efecto fractal. En M. Area (Coord.), Educar en la Sociedad de la Información (pp. 37-80). Bilbao: Desclée de Brouwer.

Sancho, J. M. (2006). De tecnologías de la información y la comunicación a recursos educativos. En J. M. Sancho (coord.), Tecnologías para transformar la educación (pp. 15-36). Madrid: Akal/Unia.

Sancho, J. M. y Alonso, C. (Coord.), La fugacidad de las políticas, la inercia de las prácticas. La educación y las tecnologías de la información y la comunicación. Barcelona: Octaedro.

Sancho, J. M. y Brain, B. (2013). Cuando la sociedad digital solo es un eco: el caso de la formación inicial de los maestros de primaria. Digital Education Review, 24, 69-82. http://greav.ub.edu/der

Sancho-Gil, J. M. y Rivera-Vargas, P. (2016). The Socio-Economic Evaluation of a European Project: The Diylab Case. Informatics, 3(13), 1 -17. doi: https://doi.org/10.3390/informatics3030013

Sancho, J, M., Giro, X.; Ornellas, A., Sánchez, J. A., Fraga, L., Guitert, M.; Alonso, K. (2012). Cuatro puntales para la mejora de la educación mediada por las TIC. En J. M. Sancho y C. Alonso (Coord.), Op. cit. (pp. 139-152).

Sancho, J. M. y Hernández, F. (1997). La investigación educativa como espacio de dilemas y contradicciones. Revista de Educación, 312, 81-110. 
Sancho, J. M. y Hernández-Hernández, F. (2014). De los por qués y los cómos de una investigación sobre el aprender a ser docente de Primaria. En J. M. Sancho y F. Hernández-Hernández (coords.), Maestros al vaivén Aprender la profesión de docente en el mundo actual (pp. 17-42). Barcelona: Octaedro.

Sancho Gil, J. M. y Padilla Petry, P. (2016). Promoting digital competence in secondary education: are schools there? Insights from a case study. Journal of New Approaches in Educational Research, 5(1), 57-63. doi: https://doi.org/10.7821/naer.2016.1.157

Sancho, J, M., Padilla Petry, P.; Domingo, L., Müller, J.; Giro, X. (2012). El Instituto La Mallola. Una apuesta por la integración de las TIC. En J. M. Sancho y C. Alonso (Coord.), Op. cit. (pp. 61-86).

Sancho-Gil, J. M., Sánchez-Valero, J. A. y Domingo-Coscollola, M. (2017). Research-based insights on initial teacher education in Spain. European Journal of Teacher Education, 40(3), 310-325 doi: https://doi.org/10.1080/02619768.2017.1320388

Sarason, S. B. (2003). El predecible fracaso de la reforma educativa. Barcelona: Octaedro.

Sawyer, K. R. (2008). Optimising Learning Implications of Learning Sciences Research. Paris: OECD.

Sennett, R. (2000). La corrosión del carácter. Las consecuencias personales del trabajo en el nuevo capitalismo. Barcelona: Anagrama

Sennett, R. (2003). Respect in a world of inequality. New York: W.W. Norton.

Sennett, R. (2006). The culture of the new capitalism. New Haven: Yale University Press.

Taylor, F. W. (1961). Principios de la administración científica. México: Herrero Hermanos.

van der Klink, M. y Boon, J. (2003). Competencies: the triumph of a fuzzy concept', International. Journal of Human Resources Development and Management, 3(2), 125-137.

Westera, W. (2001). Competences in education: A confusion of tongues. Journal of Curriculum Studies, 33(1), 75-88.

Winterton, J. (2009). Competence across Europe: highest common factor or lowest common denominator? Journal of European Industrial Training, 33(8/9), 681-700. 